\title{
On the Rearrangement of Terms in a Complex Series.
}

\author{
By Professor S. BeatTy, Toronto.
}

\section{(Received 20th February 1924. Read 7th March 1984.)}

The possibilities under rearrangement of terms in a complex series were discussed by Levy* and by Steinitz. $\dagger$ A reference to the Steinitz paper as first disposing of the questions raised was made by Bieberbach. $\ddagger$ It is the purpose of the present paper to give an independent treatment by methods somewhat resembling those of the Levy paper.

It will be supposed that there is supplied a countable set $(T)$ of numbers $T$, with or without repetitions, but with zero as the only limit point. Any scheme which sets up a one-to-one correspondence between the numbers of $(T)$ and the positive integers supplies at the same time an infinite series with the numbers of $(T)$ as terms. The set $(S)$ of all such series has the potency of the continuum, provided every two numbers of $(T)$ are regarded as distinct.

With regard to the set $(T)$, the possibilities are that the series which constitute $(S)$ are (1) all convergent, or (2) all divergent, or (3) some convergent, some divergent. Possibility (1) applies when and only when one, and therefore every, series of $(S)$ is absolutely convergent. Attention may, therefore, be directed exclusively to possibilities (2) and (3). A first problem is to show that (3) subdivides into $(3 a),(3 b)$ on the basis of whether the sums of those series of $(S)$ which are convergent constitute a straight line and no more in the complex plane, or constitute the whole complex plane. A second problem is to determine the nature of $(T)$ characteristic of (2), $(3 a),(3 b)$. These two problems will not be kept separate in what follows.

On the supposition that (1) does not apply to (T), a simple application of the Heine-Borel theorem suffices to show that there

\footnotetext{
- Nouvelles Ann. de Math., 1905.

+ Crelle's Journal, Vols. 143, 144.

\$Lehrbuch der Funktionentheorie, Vol. I, p. 20.
} 
is an amplitude $\theta$ such that, however small the positive number $\epsilon$ may be, the subset $(T, \theta, \epsilon)$ composed of all numbers of $(T)$ with amplitudes between $\theta-\epsilon$ and $\theta+\epsilon$ is one to which (1) does not apply. The numbers of $(T, \theta, \epsilon)$ are of the form

$$
e^{i \theta}(\rho \cos t+i \rho \sin t)
$$

in which $\rho>0$ and $|t|<\epsilon$. It is possible to make a selection of numbers from $(T, \theta, \epsilon)$ in such a way that the series corresponding

$$
e^{i \theta} \sum_{n} \rho_{n} \cos t_{n}, e^{i \theta} \sum_{n} i \rho_{n} \sin t_{n}
$$

are respectively divergent and absolutely convergent. These will be called respectively first-special and second-special series and their sum a special series, all relative to $(T, \theta, \epsilon)$. The special series diverges in the direction of amplitude $\theta$. It may be that there is a positive number $\epsilon^{\prime}<\epsilon$ such that, on employing all the numbers of $\left(T, \theta, \epsilon^{\prime}\right)$, the corresponding series

$$
\sum_{n} i \rho_{n} \sin t_{n}
$$

is absolutely convergent. In that case,

$$
e^{i \theta} \sum_{n} \rho_{n} \cos t_{n}, e^{i \theta} \sum_{n} i \rho_{n} \sin t_{n}
$$

are respectively first-special and second-special series, relative to $(T, \theta, \epsilon)$. If no such number $\epsilon^{\prime}$ exists, a finite number of numbers of $(T, \theta, \epsilon)$ may be selected so that the corresponding sum

$$
\Sigma_{\rho_{n}} \sin \left|t_{n}\right|
$$

is intermediate to $\frac{1}{2}$ and 1 , then a second selection of a finite number of other numbers of $\left(T, \theta, \frac{\epsilon}{2}\right)$ may be made so that the corresponding sum $\Sigma_{\rho_{n}} \sin \left|t_{n}\right|$ is intermediate to $\frac{1}{4}$ and $\frac{1}{2}$, and in general the $p^{\prime}$ th selection of a finite number of other numbers of $\left(T, \theta, \epsilon 2^{1-p}\right)$ may be made so that the corresponding sum

$$
\Sigma \rho_{n} \sin \left|t_{n}\right|
$$

is intermediate to $2^{-p}$ and $2^{1-p}$. As the result of any number of selections, the corresponding sum $\Sigma \rho_{n} \sin \left|t_{n}\right|$ is less than 4, while 
the corresponding sum $\Sigma_{\rho_{n}} \cos t_{n}$ arising from $p$ selections is greater than

$$
\frac{1}{2} \cot \epsilon+\frac{1}{4} \cot \frac{\epsilon}{2}+\ldots+\frac{1}{2^{p}} \cot \frac{\epsilon}{2^{p-1}}
$$

which increases indefinitely with $p$. Hence, in this case also, the first-special and second-special series exist.

Attention has been called to the possibility that, for every small positive number $\epsilon^{\prime}$ the series

$$
\Sigma_{\rho_{n}} \sin t_{n}
$$

may be divergent, in which the summation has reference to all numbers of $\left(T, \theta, \epsilon^{\prime}\right)$ for which $t_{n}>0$. In this case, $\theta$ is called an amplitude divergent on the left, or briefly an amplitude $D L$. In the event of convergence of the series of this type associated with some small positive number $\epsilon^{\prime}$, the amplitude $\theta$ will be said to be convergent on the left and referred to briefly as an amplitude $C L$. In the same way, $\theta$ is either an amplitude divergent on the right or convergent on the right, or briefly an amplitude $D R$ or $C R$. Either of the designations $D L, C L$ may be associated with either of $D R, C R$.

To each such amplitude $\theta$, there corresponds a point $P$ of amplitude $\theta$ on the unit circle. The set $(P)$ of such points $P$ may be finite or infinite and is closed in the latter event. Three cases arise for consideration. The lower bound of the lengths of arcs containing $(P)$ may be less than, greater than, or equal to $\pi$. These cases will be called $A, B, C$ in the order of mention above.

In case $A$, there is an arc of length less than $\pi$ containing $(P)$ within it. Denoting by

$$
e^{i(\alpha-\beta)}, e^{i(a+\beta)}
$$

the end points of this arc, it follows that all the numbers of $(T, \alpha, \beta)$ give rise, whatever be their arrangement, to a series of the form

$$
e_{n}^{i a}\left(\rho_{n} \cos t_{n}+i \rho_{n} \sin t_{n}\right),
$$

in which $\left|t_{n}\right|<\beta<\frac{\pi}{2}$ The series

$$
\Sigma_{\rho_{n},} \sum_{\rho_{n}} \cos \beta, \sum_{\rho_{n}} \cos t_{n}
$$


are all divergent. That is, the series

$$
e^{i \alpha} \sum_{n}\left(\rho_{n} \cos t_{n}+i \rho_{n} \sin t_{n}\right)
$$

is divergent. The numbers of $(T)$ which are not in $(T, \alpha, \beta)$ give rise to an absolutely convergent series. Hence, (2) applies. That is, $(S)$ contains only divergent series.

In case $B$, there are in $(P)$ three points

$$
e^{i(a-\beta)}, e^{i a}, e^{i(a+r)}
$$

such that $0<\beta, \gamma<\pi<\beta+\gamma$. Any number of amplitudes between $\alpha-\beta$ and $\alpha$ can be written in the form

$$
X e^{i(a-\beta)}+Y e^{i a} \text {, }
$$

in which $X, Y \equiv 0$. A corresponding result applies for a number in either of the two remaining divisions of the plane made by the half-lines of amplitudes $\alpha-\beta, \alpha, \alpha+\gamma$. Hence, any number can be written in the form

$$
X e^{i(a-\beta)}+Y e^{i a}+Z e^{i(a+r)}
$$

in which $X, Y, Z \equiv 0$. The numbers of $(T)$ may be divided among four series, the first three of which are sperial series with regard to

$$
(T, \alpha-\beta, \epsilon),(T, \alpha, \epsilon),(T, \alpha+\gamma, \epsilon)
$$

respectively and the fourth containing all remaining numbers of $(T)$ arranged in order of decreasing numerical value. The terms of the three first-special series taken along with the terms of the fourth series form a set called $\left(T^{\prime \prime}\right)$. Let $\Delta^{\prime}$ be any assigned number. A number $m$ can be found, such that any term after the $m^{\prime}$ th in any of the four series whose terms make up $\left(T^{\prime}\right)$ is less than an assigned positive number $\epsilon^{\prime}$. The sum of the first $m$ terms in each of the four series making up $\left(T^{\gamma}\right)$ will be denoted by $S^{\prime \prime}{ }^{\prime}$. Then, numbers $X, Y, Z \equiv 0$ exist so that

$$
X e^{i(a-\beta)}+Y e^{i a}+Z e^{i(a+\gamma)}=\Delta^{\prime}-S_{4 m}^{\prime} .
$$

Numbers $p, q, r$ can be found such that the sums of $p, q, r$ terms following the first $m$ in the three first-special series differ from

$$
X e^{i(a-\beta)}, Y e^{i a}, Z e^{i(a+\gamma)}
$$

respectively by amounts in each case less than $\epsilon^{\prime}$. Calling

it appears that

$$
4 m+p+q+r=n,
$$

$$
\left|S_{n}^{\prime}-\Delta^{\prime}\right|<3 \epsilon^{\prime}
$$


in which $S_{n}$ denotes the sum of the $n$ terms thus selected. It will be supposed that a circle of radius $\epsilon^{\prime}$ is drawn, with the origin as centre, and that there is placed outside of it a hexagon with sides parallel to the half-lines of amplitudes $\alpha-\beta, \alpha, \alpha+\gamma$, and that three of the vertices lie on these half-lines. If $S_{n}{ }_{n}-\Delta^{\prime}$ does not lie within this hexagon, a finite number of additional terms can be selected from the remainders of the three first-special series in such a way as to insure that the resulting $S_{n+j}^{\prime}-\Delta^{\prime}$ lies within the hexagon. Moreover, a finite number of additional terms can be selected from the remainders of the series making up $\left(T^{\prime}\right)$, and added in, in such a way as to insure that throughout each $S_{n+j+k}^{\prime \prime}-\Delta^{\prime}$ lies within the hexagon and the maximum absolute value of the terms remaining unselected is less than $\frac{\epsilon^{\prime}}{2}$. After this has been secured, the circle and hexagon may be replaced by two others of the same relative type but of one-half the linear dimensions. If the difference of the sum already obtained and $\Delta^{\prime}$ does not lie within the new hexagon, it suffices to add a finite number of terms from the remainders of the three first-special series to secure a difference lying in it. This property of inclusion is one that can he maintained as formerly, at the same time as the numerical value of the terms remaining unselected decreases to be less than $\frac{\epsilon^{\prime}}{4}$. The process may be continued indefinitely, by employing numbers $\epsilon^{\prime}, \frac{\epsilon^{\prime}}{2}, \frac{\epsilon^{\prime}}{4}, \frac{\epsilon^{\prime}}{8}, \ldots$ in succession. There is no number of $\left(T^{\prime}\right)$ remaining unselected, and the sum of the series so obtained is $\Delta^{\prime}$ This fact may be employed to show that $(S)$ contains a series with any assigned sum $\Delta$. For if the combined sum of the three second-special series is $D$, the numbers of $\left(T^{\prime}\right)$ may first be arranged as terms in a series having $\Delta-D$ as sum. If the terms of the second-special series are added in and each attached to the term of the first-special series from which it was separated, there results a series of $(S)$ with sum $\Delta$. The discussion has been made to depend on the utilization of three points of $(P)$. If there are more than three points in $(P)$, one might, if one wished, make use of more than three, provided the lower bound of the lengths of arcs including them exceeds $\pi$. 
In case $C$, there is a semicircular arc containing all the points of $(P)$, but the ends of the arc are points of $(P)$. Denote these end points by $e^{i a}, e^{i(a+x)}$. It may be that there is no other point in $(P)$. If so, it may happen that $a, a+\pi$ are both $C L$ and $C R$, and in that case possibility ( $3 a$ ) applies. For two series may be constructed from $(T)$, the first being the two first-special series combined into one and the second being the two second-special series and all remaining terms combined. The special series will be supposed to involve all terms of $(T, \alpha, \epsilon)$ and $(T, \alpha+\pi, \epsilon)$. The first of the two series may be arranged to have any number of the form $p^{i a}$ as sum, while the second is absolutely convergent. Denoting the sum of the second by $\sigma$, it appears that there are series in $(S)$ with any number of the form $\sigma+\rho e^{i a}$ as sum, and all the remaining series in $(S)$ are divergent. It may happen that one of $\alpha, \alpha+\pi$ is both $D L$ and $D R$, or both $\alpha, \alpha+\pi$ are $D L$, or both $a, \alpha+\pi$ are $D R$, and in this case (3b) applies. For there may be constructed from $(T)$ four series divergent in the directions of amplitudes

$$
\alpha, \alpha+\frac{\pi}{2}, \alpha+\pi, \alpha+\frac{3 \pi}{2},
$$

and these along with the remaining numbers of $(T)$ may be employed as formerly suggested to build up a series of $(S)$ with any assigned number for sum. To verify that there is a series divergent in the direction of amplitude $\alpha+\frac{\pi}{2}$, it is only necessary, in case $a$ is $D L$ for example, to consider selections made from the special series relative to $(T, \alpha, \epsilon),(T, \alpha+\pi, \epsilon)$ and also from $(T, \alpha, \epsilon)$, the numbers $t_{n}$ in the last case being positive and so related to the numbers $\rho_{n}$ that $\Sigma \rho_{n} \sin t_{n}$ is divergent The selection can be made so as to leave special series relative to $(T, \alpha, \epsilon),(T, \alpha+\pi, \epsilon)$ and to constitute a series divergent in the direction of amplitude $\alpha+\frac{\pi}{2}$, the sums of terms never being further from the half-line of amplitude $a+\frac{\pi}{2}$ than an assignable number. It may happen that a, $\alpha+\pi$, considered in one order are $C L, C R$ respectively, but considered in the opposite order there is either a $D L$ or $D R$. In that case, (2) applies. For the sum of $n$ terms in any series of $(S)$, with increase of $n$, comes to lie permanently on one side of the line 
$X e^{i a}$ and to be removed further and further from it without limit. Hence $(S)$ is made up of divergent series. But it may be that there are in $(P)$ more than the two points $e^{i a}, e^{i(a+m)}$. Denote a third point of $(P)$ by $e^{i(a+\beta)}$, where $0<\beta<\pi$, for example. It may happen that $\alpha$ is $D R$, or $\alpha+\pi$ is $D L$. In that case, possibility (3b) applies, for there may be formed four series divergent along the half-lines of amplitudes $a, \alpha+\beta, \alpha+\pi, \alpha+\frac{3 \pi}{2}$, and these along with the remaining numbers of $(T)$ may be utilized as formerly suggested to build up a series of $(S)$ with any assigned number as sum. It may happen that $\alpha$ is $C R$ and $\alpha+\pi$ is $C L$. In that case, possibility (2) applies, for the sum of $n$ terms in any series of $(S)$, with increase of $n$, comes to lie on the same side of $X e^{i a} a s e^{i(a+\beta)}$ and to be removed further and further from it without limit.

The above discussion can be simplified by employing geometrical terms to characterize the directions of amplitudes $\theta$, and their properties. Moreover, the results can be stated in terms of real variables only Suppose $(a),(b)$ are countable sets of real numbers with zero as their only limit point. Suppose a one-to-one correspondence is established and maintained between the numbers of the two sets The result has reference to the Cartesian points $(x, y)$ which it is possible to represent, in denoting by $x$ the sum of the numbers of (a), written in series according to some law of arrangement, and by $y$ the sum of the numbers of $(b)$, written in series according to the arrangement implied by that applied to (a). From this point of view, the discussion can be extended to higher dimensions. 